\title{
Hematomas epidurales bilaterales simultáneos
}

\author{
M. Gelabert-González; M. Iglesias-País; R. Serramito-García; J. Fernández-Villa; A. García-Allut y R. Martínez- \\ Rumbo
}

Servicio de Neurocirugía. Hospital Clínico Universitario de Santiago. Departamento de Cirugía. Universidad de Santiago de Compostela.

\section{Resumen}

Introducción. Los hematomas epidurales constituyen una de las complicaciones más frecuentes de los traumatismos craneales. Los hematomas bilaterales son muy poco frecuentes, se asocian a traumatismos de gran intensidad y se acompañan de una elevada mortalidad.

Objetivos. Analizar los 6 casos de hematoma epidural bilateral (HEB) tratados en los últimos 24 años que representan el $2.5 \%$ de todos los hematomas epidurales intervenidos quirúrgicamente en el mismo período de tiempo.

Resultados. La serie esta formada por 5 varones y 1 mujer con una edad media de 32.6 años (rango 16-55). Clínicamente todos presentaban disminución del nivel de consciencia. En 3 pacientes el hematoma sobrepasaba la línea media, y en los otros 3 las localizaciones eran diversas. Se apreció fractura de cráneo en la totalidad de los pacientes. Todos los hematomas fueron tratados quirúrgicamente y la mortalidad operatoria fue del $50 \%$.

Conclusiones. El hematoma epidural bilateral es una entidad clínica poco frecuente y cuyo pronóstico depende fundamentalmente de la situación neurológica preoperatoria.

PALABRAS CLAVE: Fractura de cráneo Hematoma epidural bilateral. Tomografía computarizada. Traumatismo craneoencefálico.

Simultaneous bilateral extradural haematoma

\section{Summary}

Introduction. Epidural haematomas are one of the most common complications of closed head injuries. Bilateral extradural haematomas are rare, usually acute, and generally associated with severe trauma and a high mortality.

Recibido: 24-06-04. Aceptado: 18-10-04
Objective. The purpose of this paper is to present six cases of bilateral extradural haematomas seen at our service during the last $\mathbf{2 4}$ years and found a incidence of $\mathbf{2 . 5 \%}$ of all cases of extradural haematomas surgically treated.

Results. There were 5 males and 1 female with a mean age of 32.6 years (range 16-55). In 3 cases haematoma was across the midline and in the other 3 cases haematomas was found at different locations on cither side. Skull fracture was present in all cases. Surgical approach was the primary treatment in all cases. Mortality in our series was $\mathbf{5 0 \%}$.

Conclusions. Bilateral extradural haematomas is a rare condition and the prognosis is mainly dependent of the pre-operative neurological state.

KEY WORDS: Bilateral epidural haematoma. Head injury. Computed tomography. Skull fracture.

Introducción

Los hematomas epidurales (HE) constituyen una complicaciónfrecuentedelos traumatismos craneoencefálicos ${ }^{15,17,20}$. Afectan por lo general a pacientes jóvenes y tienen una mayor incidencia en la región temporal ${ }^{14,19}$. Habitualmente su localización es unilateral, habiéndose publicado casos bilaterales cuya presentación puede ser simultánea ${ }^{1,5,17} \mathrm{o}$ sucesiva $^{4}$. En otras ocasiones, los HE aparecen como complicación de procedimientos quirúrgicos previos realizados en su cercanía o a distancia ${ }^{8}$ que, excepcionalmente, también pueden ser bilaterales ${ }^{22,23}$.

Presentamos nuestra experiencia con 6 casos de HEB de presentación simultánea atendidos en los últimos 24 años.

Pacientes y métodos

Realizamos un estudio retrospectivo de las historias

Abreviaturas. HE: hematoma epidural. HEB: hematoma epidural bilateral. GCS: Glasgow coma scale. GOS: Glasgow outcome scale. 

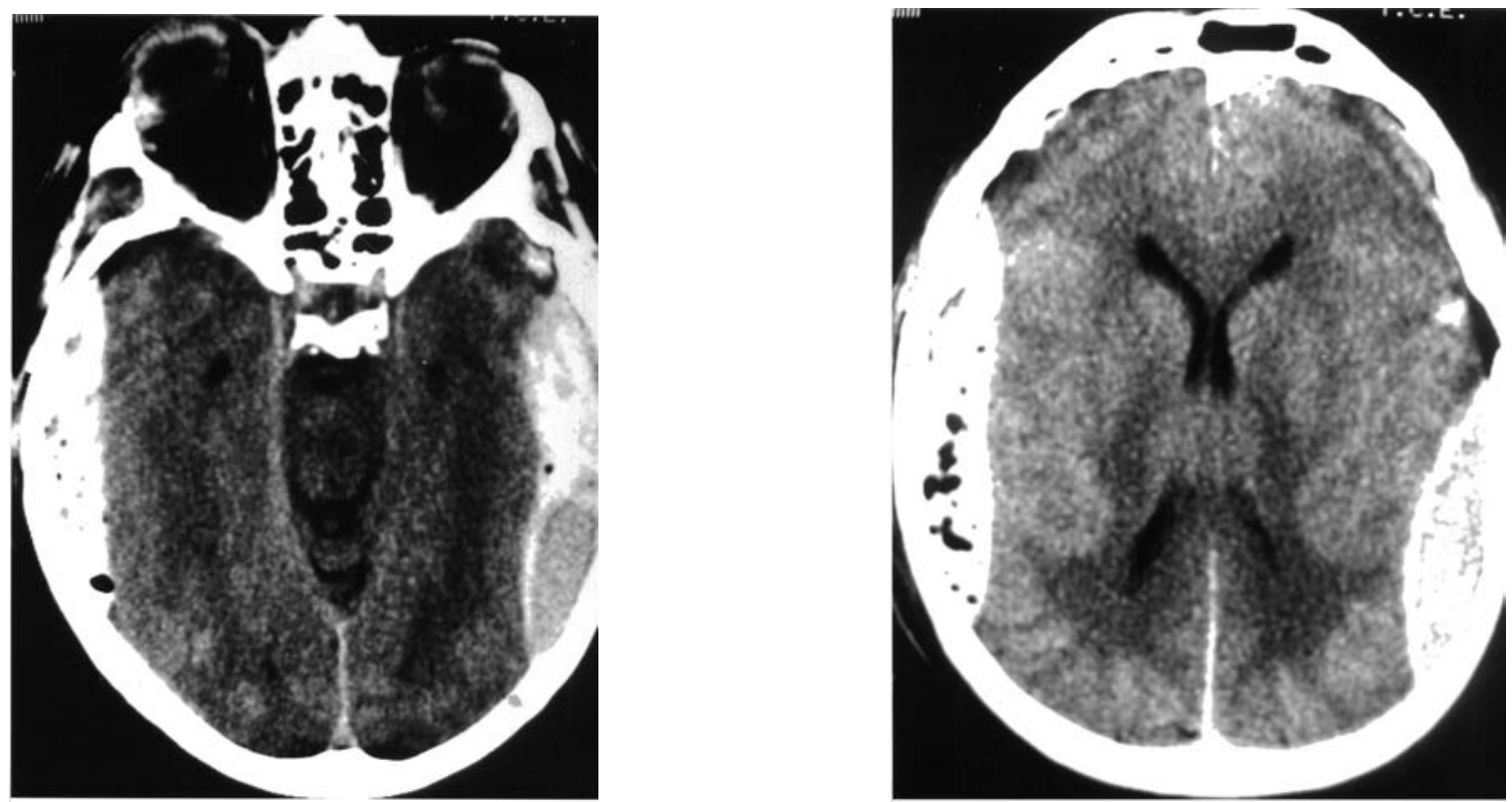

Figura 1. Tomografia computarizada (caso 2). Hematoma epidural bilateral temporo-parietal bilateral.
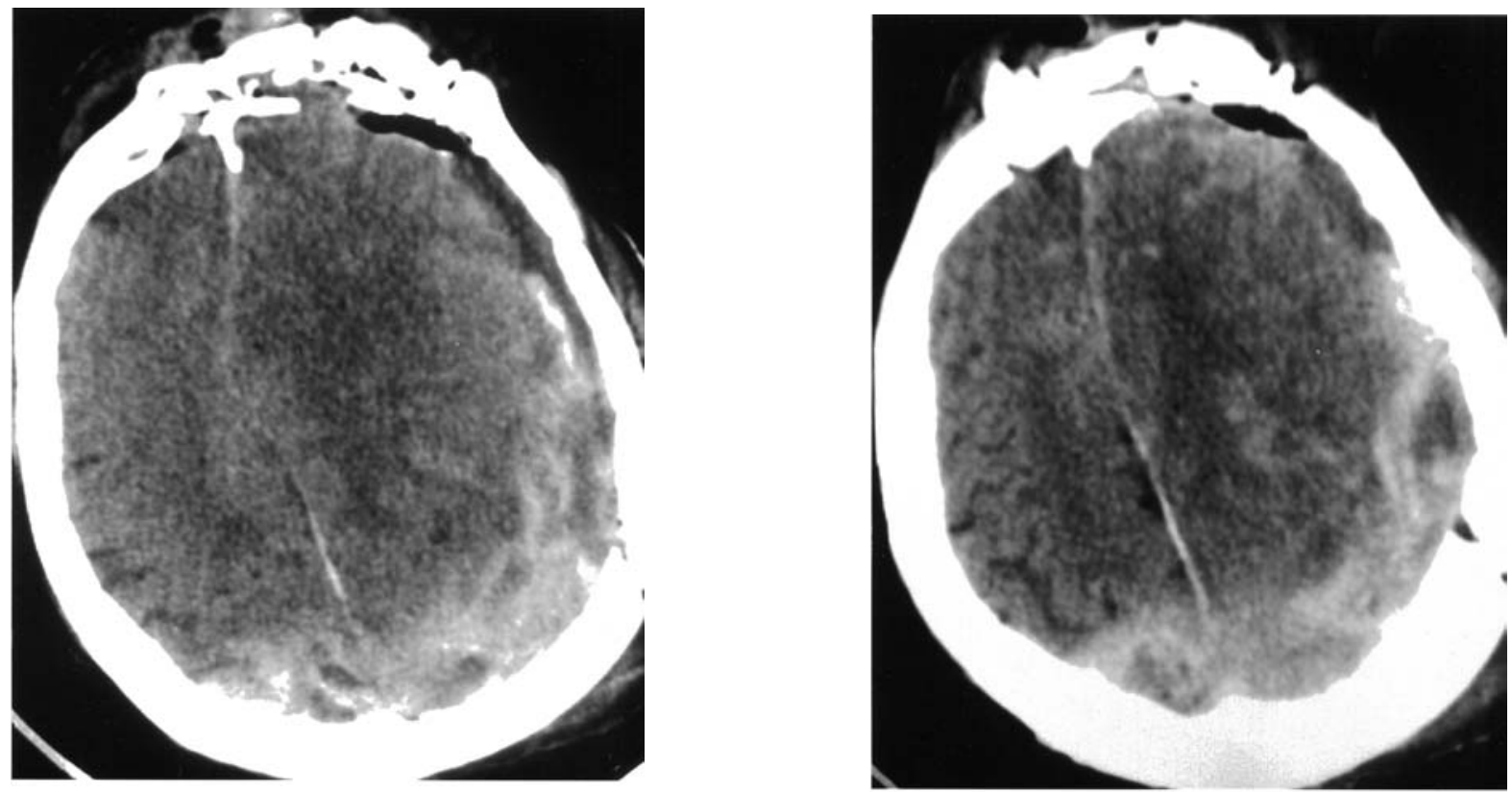

Figura 2. Tomografía computarizada (caso 6). Fracturas craneales múltiples. Hematoma epidural parietal bilateral que cruza la línea media.

clínicas de 6 pacientes intervenidos quirúrgicamente de un hematoma epidural bilateral en un período de 24 años. Se revisaron la edad y sexo de los pacientes, el intervalo transcurrido desde el traumatismo hasta el diagnostico/tratamiento, el nivel de conciencia de acuerdo con la escala de coma de Glasgow (GCS), la existencia o no de fractura craneal $\mathrm{u}$ otras lesiones asociadas obteniendo estos datos de la revisión de los estudios de imagen y/o del informe operatorio, el tratamiento realizado y la evolución de los pacientes de acuerdo con la escala de resultados de Glasgow (GOS). Dos casos fueron publicados previamente como notas clínicas ${ }^{9,10}$.

\section{Resultados}

Entre los años 1980 y 2003 hemos atendido un total de 
Tabla 1

Características clínico-radiológicas de los pacientes

\begin{tabular}{|c|c|c|c|c|c|c|c|}
\hline Caso & $\begin{array}{l}\text { Edad } \\
\text { Sexo }\end{array}$ & $\begin{array}{l}\text { Intervalo } \\
\text { trauma- } \\
\text { diagnóstico }\end{array}$ & GCS & Lesiones derechas & Lesiones izquierdas & Hematoma & GOS \\
\hline 1 & $23 / \mathrm{V}$ & $7 \mathrm{~h}$ & 11 & Fractura temporal & Fractura temporo-parietal & Temporal bilateral & 5 \\
\hline 2 & $28 / \mathrm{V}$ & $1 \mathrm{~h}$ & 7 & Fractura temporo-parietal & No & Temporo-parietal bilateral & 5 \\
\hline 3 & $31 / \mathrm{V}$ & $3 \mathrm{~h}$ & 10 & Fractura temporo-parietal & Fractura parietal & $\begin{array}{l}\text { Temporo-parietal derecho } \\
\text { Parietal izquierdo }\end{array}$ & 5 \\
\hline 4 & $16 / \mathrm{V}$ & $2 \mathrm{~h}$ & 14 & Estallido craneal & Estallido craneal & Frontal bilateral & 11 \\
\hline 5 & $55 / \mathrm{V}$ & $2 \mathrm{~h}$ & 5 & $\begin{array}{l}\text { Fractura occipital } \\
\text { Contusiones en lóbulos }\end{array}$ & $\begin{array}{l}\text { Fractura occipital } \\
\text { Contusión frontal } \\
\text { frontal y temporal }\end{array}$ & Occipital bilateral & 1 \\
\hline 6 & $43 / \mathrm{M}$ & $4 \mathrm{~h}$ & 5 & Estallido craneal & Estallido craneal & Parietal bilateral & 1 \\
\hline
\end{tabular}

GCS: Glasgow coma scale; GOS: Glasgow outcome scale

311 pacientes afectados de hematoma epidural intracraneal de origen traumático, de los cuales 233 fueron intervenidos quirúrgicamente. De estos pacientes, seis $(2.5 \%)$ presentaban un hematoma epidural bilateral (Tabla 1).

Las edades oscilaron entre 16 y 55 años (media 32.6), siendo 5 varones y 1 mujer. El intervalo calculado aproximadamente entre el traumatismo y el diagnóstico/ tratamiento osciló entre 1 y 7 horas (media 3,1), siendo de 2,6 horas en los fallecidos y de 3,6 en los supervivientes. Los pacientes presentaban una puntuación en la escala de Glasgow entre 4 y 11.

En todos los casos se evidenció fractura craneal en relación con el hematoma. En los casos $\mathrm{N}^{\circ} 3,4$ y 6 la línea de fractura rebasaba la línea media craneal sobre la sutura sagital. La localización de los hematomas aparece recogida en la Tabla 1. (Figs. 1 y2).

Todos los hematomas fueron evacuados quirúrgicamente, evidenciándose en 5 casos su origen en arterias meníngeas, en 4 su relación con desgarro de senos venosos y en los otros 3 el sangrado procedía del díploe.

La tasa de mortalidad fue del $50 \%$ y en los pacientes que murieron la puntuación inicial en la escala de Glasgow era inferior a 6 . Los otros 3 pacientes se recuperaron $\sin$ secuelas.

\section{Discusión}

Los hematomas epidurales intracraneales son habitualmente unilaterales siendo poco frecuente los que se presentan de forma bilateral. La primera intervención sobre un hematoma epidural bilateral fue realizada por James Hill en 1772 con la recuperación completa del paciente ${ }^{1}$. El segundo caso publicado corresponde a un accidentado intervenido por G.C. Roy en 1884 de un hematoma epidural izquierdo, que falleció, apreciándose en la autopsia un hematoma en el lado derecho ${ }^{18}$.

En nuestra experiencia los HEB constituyen el 2.5\% de los HE intervenidos quirúrgicamente, representando en la mayoría de las series entre 2 y $15 \%$ de todos los hematomas epidurales del adulto ${ }^{1,7,11,13}$, llegando hasta el $22.4 \%$ en la casuística de Subrahmanian et al. ${ }^{21}$ y a $24.6 \%$ en la serie de Bozbuga et $\mathrm{al}^{3}$, aunque esta última recoge únicamente hematomas epidurales de la fosa craneal posterior. Para algunos autores son excepcionales en la infancia ${ }^{7,12}$ aunque en la experiencia de Dharker y Bhargava llegan a suponer hasta el $20 \%{ }^{6}$.

Clínicamente la pérdida de conciencia es más importante en los HEB que en los unilaterales. En nuestra experiencia, solamente 2 pacientes (33.4\%) tenían más de 8 puntos en la escala de Glasgow. Frank et $\mathrm{al}^{7}$ encontraron que solamente $2 \%$ de sus casos no estaban en coma, sin embargo en la serie de Dharker y Bhargava ${ }^{6}$ existe un contraste en este aspecto ya que únicamente el $30 \%$ de sus pacientes tenían menos de 8 puntos en la escala de Glasgow, asi mismo el $26.3 \%$ de los casos estaban en coma en la serie de Görgülü et $\mathrm{al}^{11}$.

Habitualmente se trata de hematomas simétricos que cruzan la línea media en la región parietal u occipital. Su 
desarrollo es en muchas ocasiones subagudo o diferido y pueden presentarse de dos formas diferentes: simultáneos en el tiempo ${ }^{1}$, o más frecuentemente sucesivos ${ }^{4}$.

Para la aparición de un hematoma epidural es necesario que la duramadre se separe del hueso, por lo que en este tipo de hematomas bilaterales, esta separación debe producirse en dos puntos separados entre sí. Frank et $\mathrm{al}^{7}$, en su revisión de la literatura sobre HEB, demuestran que la dirección más común de la fuerza del traumatismo que produce un HEB lleva una dirección antero-posterior en el $75 \%$ de los casos y lateral en el $25 \%$, al contrario de lo que ocurre con los hematomas unilaterales en los que la fuerza del impacto actúa en el 80\% de los casos en dirección lateral. Esta teoría apoyaría la mayor incidencia de hematomas epidurales bilaterales en la región frontal. Sin embargo, esta hipótesis puede ser cierta en hematomas subagudos o diferidos, ya que en los HEB de presentación aguda, las fuerzas del traumatismo actúan en varias direcciones provocando fracturas craneales múltiples y secundariamente los hematomas.

El origen del sangrado en los HEB es generalmente venoso $^{7}$. Así en la serie de 20 casos de Dharker y Bhargava ${ }^{6}$ no se observó en ningún caso sangrado de origen arterial. En nuestra experiencia 5 casos $(41.6 \%)$ tenían un sangrado claramente arterial.

Barlow y $\mathrm{Kohi}^{2}$ describen 2 tipos de HEB. En el primero, quizás el más frecuente, uno o los dos hematomas son diferidos y tienen un origen venoso. El segundo tipo, como el que ellos publican, los dos hematomas son de origen arterial y se presentan inmediatamente tras el traumatismo. Estos mismos autores plantean la duda sobre qué hematoma se debe tratar en primer lugar, el de mayor volumen o aquél que asienta sobre el área más elocuente. Concluyen que les parece más oportuno operar primero el hematoma asentado sobre el hemisferio dominante, aunque lo ideal sería operar los dos hematomas de forma simultánea lo cual no siempre es posible con facilidad, si exceptuamos los hematomas frontales u occipitales.

El pronóstico general del los HE es bueno con cifras de mortalidad que, en la mayoría de las veces, están por debajo del $10 \%$, aún en pacientes operados en coma ${ }^{20}$. En los HEB el pronóstico no es tan afortunado y las cifras oscilan entre el 15 y $20 \%{ }^{6,11,13}$. En nuestra experiencia la mortalidad fue considerablemente más elevada que en las series revisadas, motivada fundamentalmente por el grave deterioro neurológico que presentaban estos pacientes (puntuaciones en la escala de Glasgow de 4 y 5) y la existencia de graves lesiones asociadas, tanto craneales como encefálicas en el grupo de los pacientes fallecidos.

\section{Bibliografía}

1. Arienta, C., Baiguini, M., Granata, G., Villani, R.: Acute bilateral epidural hematomas. Report of two cases and review of the literature. J Neurosurg Sci 1986; 30: 139-142.

2. Barlow, P., Kohi, Y.M.: Acute simultaneous bilateral extradural hematoma. Surg Neurol 1985; 23: 411-413.

3. Bozbuga, M., Izgi, N., Polat, G., Gürel, 1.: Posterior fossa epidural haematomas: observations on a series of 73 cases. Neurosurg Rev 1999; 22: 34-40.

4. Burbridge, B.: Asynchronous bilateral epidural hematomas. Can Assoc Radiol 1993; 44: 390-392.

5. d'Avella D, De Blasi F, Frattarelli M, Santero G, del Vivo RE.: Acute bilateral extradural haematoma in an adult. Neurochirurgia (Stuttg) 1985; 28: 178-179.

6. Dharker, S.R., Bhargava, N.: Bilateral epidural haematoma. Acta Neurochir (Wien) 1991; 110: 29-32.

7. Frank, E., Berger, T.S., Tew, J.M.Jr.: Bilateral epidural hematoma. Surg Neurol 1982; 17: 218-222.

8. Gelabert, M., Bollar, A., García, A., Prieto, A.: Hematoma epidural como complicación de una válvula ventrículoperitoneal. Presentación de un caso y revisión de la literatura. Rev Neurol 1987; 76: 189-192.

9. Gelabert, M., Prieto, A., Rumbo, R.M., Allut, A.G.: Simultaneous bilateral extradural haematoma. Br J Neurosurg 1993; 7: 95-97.

10. Gelabert, M., Prieto, A., Allut, A.G.: Acute bilateral extradural haematoma of the posterior craneal fossa. Br J Neurosurg 1997; 11: 573-575.

11. Görgülü, A., Çobanoğlu, S., Armağan, S., Karabağli, H., Tevrüz, M.: Bilateral epidural hematoma. Neurosurg Rev 2000; $23: 30-33$.

12. Grabel, J.C., Sacher, M., Rothman, A.S.: Bilateral extradural haematomas in a thrombocytopenic infant receiving anticoagulants: case report. Neurosurgery $1989 ; 25$ : 828830 .

13. Gupta, S.K., Tandon, S.C., Mohanty, S., Asthana, S., Sharma, S.: Bilateral traumatic extradural haematomas: report of 12 cases with a review of the literature. Clin Neurol Neurosurg 1992; 94: 127-131.

14. Herrera, E.J., Viano, J.C., Aznar, I.L., Suárez, J.C.: Postraumatic intracraneal hematomas in infancy. A 16-year experience. Childs Nerv Syst 2000; 16: 585-589.

15. Ramzan, A., Wani, A., Malik, A.H., Kirmani, A., Wani, M.A.: Acute bilateral extradural hematomas. Neurol India 2002; 50: 217-219.

16. Rivas, J.J., Lobato, R.D., Sarabia, R., Cordobés, F., Cabrera, A., Gómez, P.: Extradural hematoma: Análisis of factors influencing the courses of 161 patients. Neurosurgery 1988; 23: 44-51.

17. Rochat, P., Johannensen, H.H., Poulsgard, L., Bogeskov, L.: Sequentially evolved bilateral epidural haematomas. Clinical Neurol Neurosurg 2002; 105: 39-41. 
18. Roy, G.C.: Fracture of skull, extensive extravasation of blood on dura mater, producing compression of brain; trephining; partial relief of symptoms; death. Lancet 1884; 2: 319.

19. Servadei, F., Vergoni, G., Staffa, G., Zappi, D, Nasi, M.T., Donati, R, Arista, A.: Extradural haematomas: How many deaths can be avoided. Protocol for early detection of haematoma in minor head injuries. Acta Neurochir (Wien) 1995; 133: 50-55.

20. Servadei, F.: Prognostic factors in severely head injured adult patients with epidural haematomas. Acta Neurochir (Wien) 1997; 139: 273-278.

21. SubrahmanianM.V., Rajendraprasad, G.B., Rao, B.D.: Bilateral extradural haematomas. Br J Surg 1975; 62: $397-$ 400.

22. Suzuki, S., Sayito, K., Iwabuchi, T., Hayashi, A.: Bila- teral supratentorial epidural haematomas occuring independently and consecutively alter the removal of a craniospinal meningioma. Acta Neurochir (Wien) 1982; 65: 61-66.

23. Wolfsberger, S., Gruber, A., Czech, T.: Multiple supratentorial epidural haematomas after posterior fossa surgery. Neurosurg Rev 2004; 27: 128-132.

Gelabert-González, M.; Iglesias-País, M.; SerramitoGarcía, M.; Fernández-Villa, J.; García-Allut, A.; MartínezRumbo, A.: Hematomas epidurales bilaterales simultáneos. Neurocirugía 2005; 16: 256-260.

Correspondencia postal: Miguel Gelabert González. Servicio de Neurocirugía. Hospital Clínico. La Choupana.15706 Santiago de Compostela 